\title{
Characterization of Yb-doped silica optical fiber as real-time dosimeter
}

Ivan Veronese

Norberto Chiodini

Simone Cialdi

Eduardo D'Ippolito

Mauro Fasoli

Salvatore Gallo

Eleonora Mones

Anna Vedda

Gianfranco Loi 


\title{
Characterization of Yb-doped silica optical fiber as real-time dosimeter
}

\author{
Ivan Veronese*a,b, Norberto Chiodini ${ }^{\mathrm{c}}$, Simone Cialda ${ }^{\mathrm{a}, \mathrm{b}}$, Eduardo D'Ippolito ${ }^{\mathrm{a}}$, Mauro Fasoli ${ }^{\mathrm{c}}$, \\ Salvatore Gallo ${ }^{\mathrm{a}, \mathrm{b}}$, Eleonora Mones ${ }^{\mathrm{d}}$, Anna Vedda ${ }^{\mathrm{c}}$, Gianfranco Loi ${ }^{\mathrm{d}}$ \\ ${ }^{\mathrm{a}}$ Department of Physics, Università degli Studi di Milano, Milano, Italy; ${ }^{\mathrm{b}}$ National Institute of \\ Nuclear Physics (INFN), Section of Milan, Milano, Italy; 'Dipartimento di Scienza dei Materiali, \\ Università degli Studi di Milano Bicocca, Milano, Italy; ${ }^{\mathrm{d}}$ Medical Physics Department, Azienda \\ Ospedaliera Maggiore della Carità, Novara, Italy
}

\begin{abstract}
The near-infrared radioluminescence and dosimetric properties of Yb-doped silica optical fibers, coupled with an optical detector prototype based on an avalanche photo-diode, were studied by irradiating the fibers with clinical beams generated by a Varian Trilogy accelerator. The performances of the system in standard and small field sizes have been also investigated comparing the output factor, percent depth dose and off axis ratio measurements of the prototypal dosimetric system with other commercial sensors.

The results demonstrated that the drawback due to the stem effect in Yb-doped silica optical fibers can be managed in a simple but effective way by optical filtering. These features, together with the accuracy and precision achieved by $\mathrm{Yb}$ doped fibers in relative dose assessments make the device promising for in-vivo dosimetry studies in radiation therapy.
\end{abstract}

Keywords: Ytterbium, optical fiber, radioluminescence, Cerenkov, dosimetry, doped silica, radiotherapy

\section{INTRODUCTION}

In the last few years, there has been significant development of radiation therapy (RT) machines. Common features of these modern delivery systems are the modulation of the radiation beam, the use of radiation fields of small dimensions ${ }^{1)}$ suitable for stereotactic treatments and the high conformation of the dose to the target with the consequent possibility of dose escalation and hypo-fractionation approaches ${ }^{2}$.

All these improvements could be a benefit for the patient, but at the same time open new challenges in terms of patient safety $^{3-5)}$, of treatment planning strategies, as well as of measurements ${ }^{6-8)}$. In fact, instruments and procedures that were suitable up to few years ago now may fail or provide results not sufficiently accurate ${ }^{9}$.

In this contest, scintillating optical fiber dosimeters could be particularly useful in various applications ${ }^{10-16)}$. Essentially, such dosimeter consists in a small scintillator that originates a radioluminescent (RL) signal when exposed to ionizing radiation. The scintillator is connected to an optical fiber acting as light guide to a suitable optical detector. In principle, an optical fiber dosimeter should enable the real time measurement of the dose rate in a specific point of the radiation field ${ }^{17)}$. The small dimensions could be exploited in small field dosimetry and in-vivo dosimetry. Moreover, the RL signal is unaffected by magnetic field, and so it could be particularly useful for dosimetry measurements in the new hybrid MRI-LINAC systems.

*ivan.veronese@unimi.it; phone+390250317432

Hard X-Ray, Gamma-Ray, and Neutron Detector Physics XIX, edited by Arnold Burger, Ralph B. James,

Michael Fiederle, Larry Franks, Stephen A. Payne, Proc. of SPIE Vol. 10392, 103921D

(c) 2017 SPIE · CCC code: 0277-786X/17/\$18 - doi: 10.1117/12.2272573 
Actually, the interest in these systems dates back to the early ' $90{ }^{18)}$, but it is only almost twenty years later that the first commercial system became available, the Exradin W1 by Standard Imaging ${ }^{19)}$. The main reason of this long history was the need to face efficiently the intrinsic drawback of fiber optic dosimeters, which is the so-called stem effect. The stem effect is an additional luminescent signal due to the irradiation of the optical fiber that contributes mainly in terms of Cerenkov light. The stem effect is a spurious signal since it strongly depends on the irradiation conditions, typically the length of fiber directly exposed to the radiation beam and the direction of incidence of the beam on the fiber axis.

Various methods for the correction or subtraction of the stem effect have been proposed in the literature ${ }^{20-24)}$. Unfortunately, all of them have showed some limits in various applications and therefore new solutions are welcome. In particular, it is clear that the attractiveness of fiber optic dosimeters would be greatly enhanced by employing a scintillation signal free from any spectral superposition with the spurious ones. We demonstrated that it is possible using a scintillator with an emission in the near infrared, that can be clearly distinguished from the stem effect emission occurring in the UV-VIS spectral region ${ }^{25,26)}$. This work aims to summarize the main findings of a recent comprehensive characterization of the dosimetric properties of $\mathrm{Yb}$-doped silica optical fibers coupled with an optical detector prototype based on an avalanche photo-diode (APD) ${ }^{27)}$.

\section{MATERIALS AND METHODS}

\subsection{The dosimetric system}

A picture of the dosimetric system is shown in figure 1. The scintillator consisted in a small portion of Yb-doped silica optical fiber with diameter of approximately $200 \mu \mathrm{m}$ and length $3 \mathrm{~mm}$, prepared by sol-gel and "rod in tube" technique. Details of the synthesis procedure of doped-silica matrices are available elsewhere ${ }^{28}$. The doped portion was connected by fusion spicing with a $1 \mathrm{~m}$ long commercial optical fiber (Polymicro Technologies, AZ, USA).

A $15 \mathrm{~m}$ long multimode optical fiber extension (FT200EMT, Thorlabs Inc., NJ, USA) was used to connect the scintillating fiber, positioned in the treatment room where the medical linear accelerator (LINAC) was installed, to the optical detector localized outside the room. On the way to reach the detector, the RL signal was optically filtered by a series of two long-pass filters with a cut-on wavelength of $950 \mathrm{~nm}$ (FELH0950, Thorlabs) using an inline filter holder (B\&W Tek Inc., DE, USA). SMA 905 type connectors achieved all the connections among the various optical components.

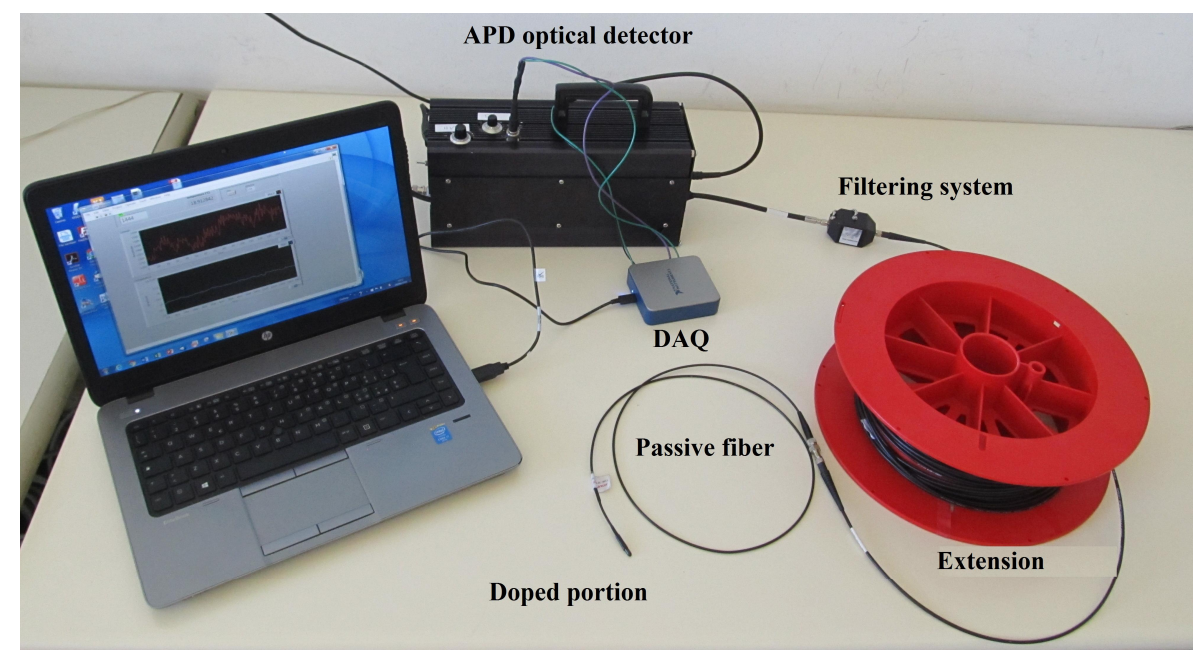

Figure 1. Picture of the fiber optic based dosimetric system. 
The optical detector was a laboratory-made photon counting system based on a silicon APD (C309021S, Perkin Elmer Optoelectronics, Canada) operating in Geiger mode. The APD was characterized by a quantum efficiency (QE) curve extending beyond $1000 \mathrm{~nm}$, with typical QE values of $13 \%$ at a wavelength around $975 \mathrm{~nm}$, where the $\mathrm{Yb}^{3+}$ emission occurs. The package of the detector chip contained a light-pipe which fell within the active area of the detector, allowing efficient coupling with the optical fiber. The APD was cooled down to a temperature of approximately $-19{ }^{\circ} \mathrm{C}$ by means of a two-stage Peltier thermoelectric cooling system. A specific circuit was implemented to quench and digitalize the APD breakdown pulse. A data-acquisition device (DAQ USB-6000, National Instruments, TX, USA) connected to a personal computer was finally used to acquire the signals of interest.

\subsection{The irradiations}

The irradiations of the fibers were carried out with $6 \mathrm{MV}$ X-rays in Flattering Filter Free (FFF) modality, and $9 \mathrm{MeV}$ electron beams generated by a Varian Trilogy TX accelerator (Varian Medical Systems Inc., CA, USA).

According to the specific experimental set up, the fiber was placed in a Solid Water ${ }^{\mathrm{TM}}$ phantom (GAMMEX rmi, WI, USA) or in a motorized water phantom (Blue Phantom, IBA Dosimetry GmbH, Germany). The various irradiation conditions and geometries used for the tests are described in detail elsewhere ${ }^{27)}$.

A picture of the medical LINAC used for the irradiations and of a typical experimental set up is shown in figure 2 .
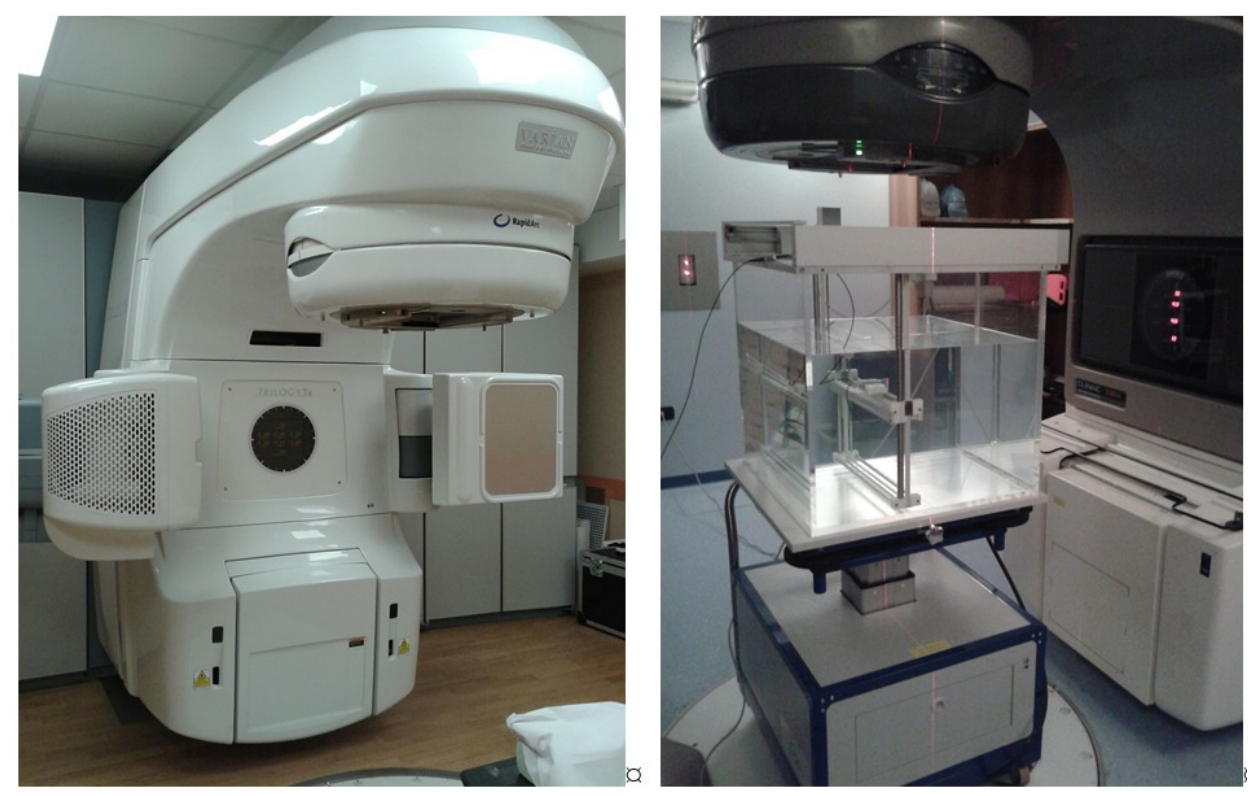

Figure 2. Varian Trilogy TX accelerator and the motorized water phantom used for the irradiation of the fibers.

First, the general dosimetric properties of the system like sensitivity, reproducibility, dose response and dose-rate dependence were tested. Afterwards, the efficacy of the optical filtering approach for the stem effect removal was evaluated.

Finally, the performance of the system in standard and small field sizes were investigated, comparing the measurements obtained for the output factors (OFs), percentage depth dose (PDD) and off-axis ratio (OAR) of the prototype with those of other commercial detectors, including the Exradin W1 plastic scintillator. 


\section{RESULTS AND DISCUSSION}

The dosimetric system, tested under clinical conditions, showed a satisfactory sensitivity, reproducibility, and a linear dose-rate response. A reliable dose evaluation was obtained independently of the dose rate and of the orientation of the impinging beam, clearly demonstrating that stem signal (and, more specifically, its Cherenkov component) was very efficiently suppressed ${ }^{27)}$.

Furthermore, the results showed a good agreement with reference dosimeters in terms of relative dose profiles and output factors. Examples of such measurements are shown in figures 3, 4 and 5. Figure 3 show the PDD obtained with the Ybfiber compared with the reference one, measured by using a CC13 ionization chamber (IBA Dosimetry GmbH, Germany). The deviations between the two dosimeters were negligible at any depth and within the fiber reproducibility error (i.e. approximately $1 \%$ ).

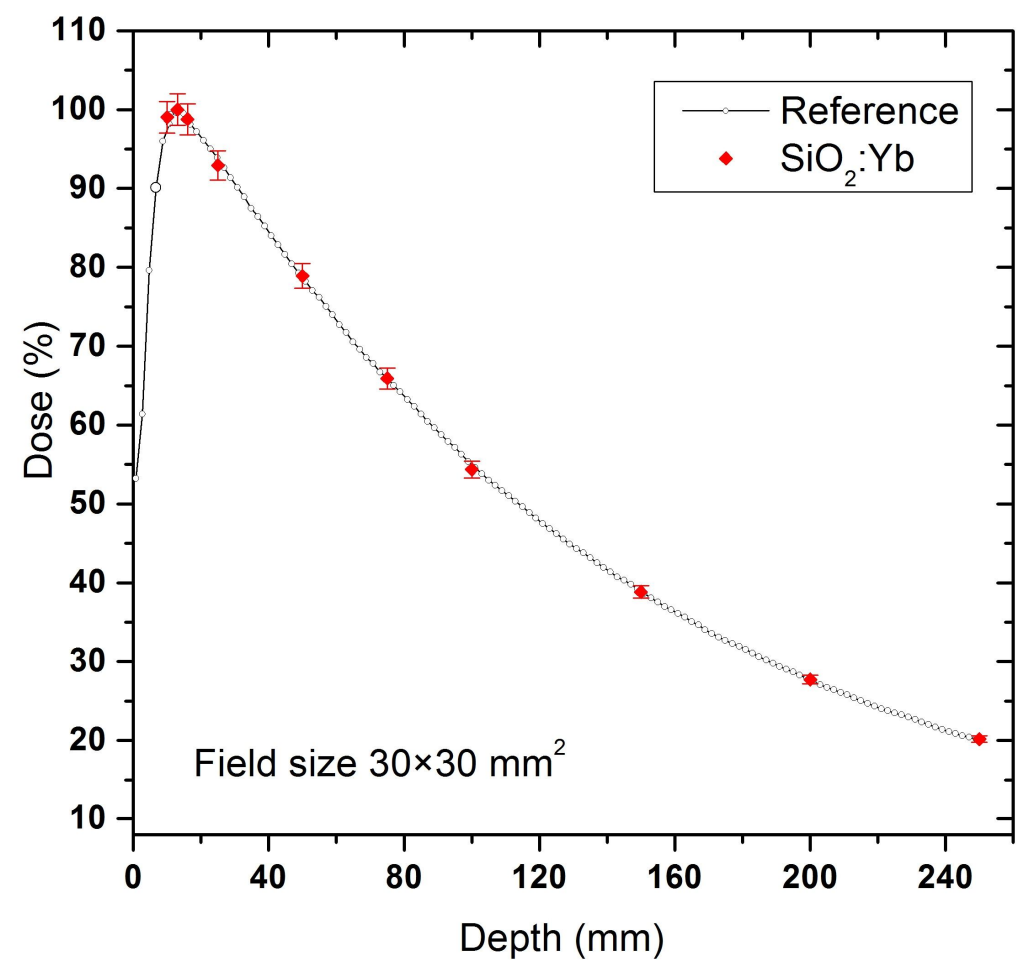

Figure 3. Example of Percentage Depth Dose (PDD) curve in water for a 6 MV FFF X-ray field with size $30 \times 30 \mathrm{~mm}^{2}$ obtained with the $\mathrm{Yb}$-doped fiber compared with that measured by a CC13 ionization chamber, used as reference.

Figure 4 shows the OAR of the field $6 \times 6 \mathrm{~mm}^{2}$ obtained with the Yb-doped silica optical fiber and with the commercial scintillator Exradin W1. These results are compared with the reference profile obtained by means of radiochromic films EBT3. The data point of figure 4 were centred in the inflection point of the penumbra region.

The agreement between the prototype detector and the EBT3 film profiles was good for all of the investigated fields with maximum deviation in the high-gradient-region less than 5\% and within the detector reproducibility in low-gradient-high and low-dose-regions. For the smallest field size investigated (i.e. the OAR of figure 4) the penumbra regions resulted slightly broader than the reference one, but they were in good agreement with the profiles obtained by the commercial fiber W1. It suggests that such differences could be due to a partial volume effect, having both the scintillators a similar length $(3 \mathrm{~mm})$. No significant dependence from the energy due to not water equivalence of the $\mathrm{Yb}$-fiber was observed. 


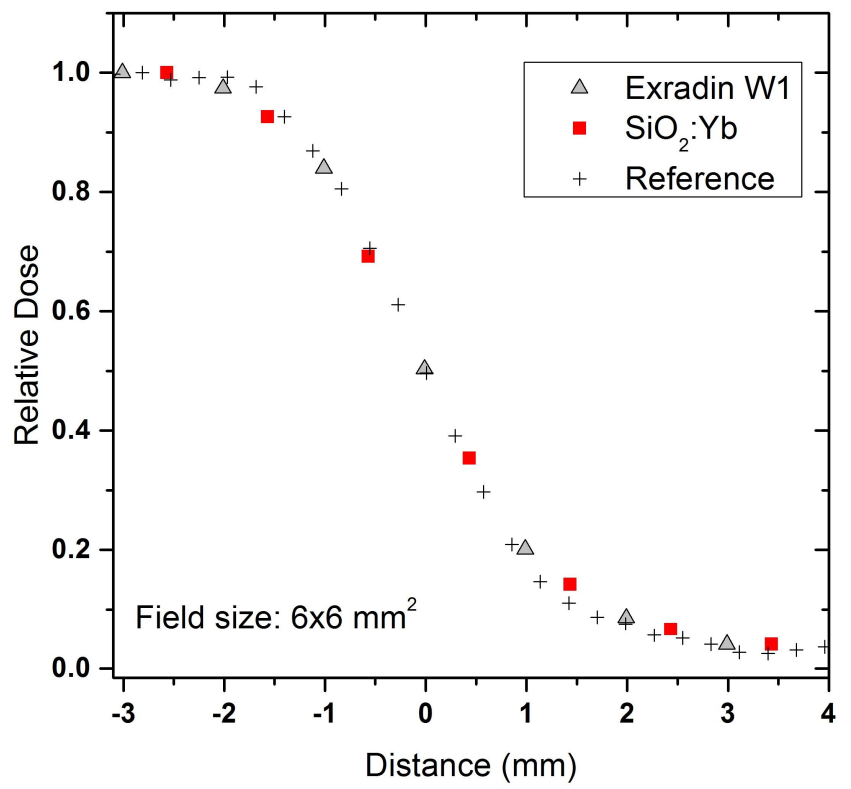

Figure 4. Example of off axis ratio (OAR) in water for a $6 \mathrm{MV}$ FFF X-ray field with size $6 \mathrm{x} 6 \mathrm{~mm}^{2}$ obtained with the Ybdoped fiber and with the commercial scintillator Exradin W1, compared with the results of radiochromic films EBT3, used as reference.

Figure 5 shows the OFs of a 6 MV FFF X-ray beam measured with the Yb-doped fiber and compared with those obtained by the commercial scintillator Exradin W1. The agreement between the two detectors was within $1 \%$ independently of the field size.

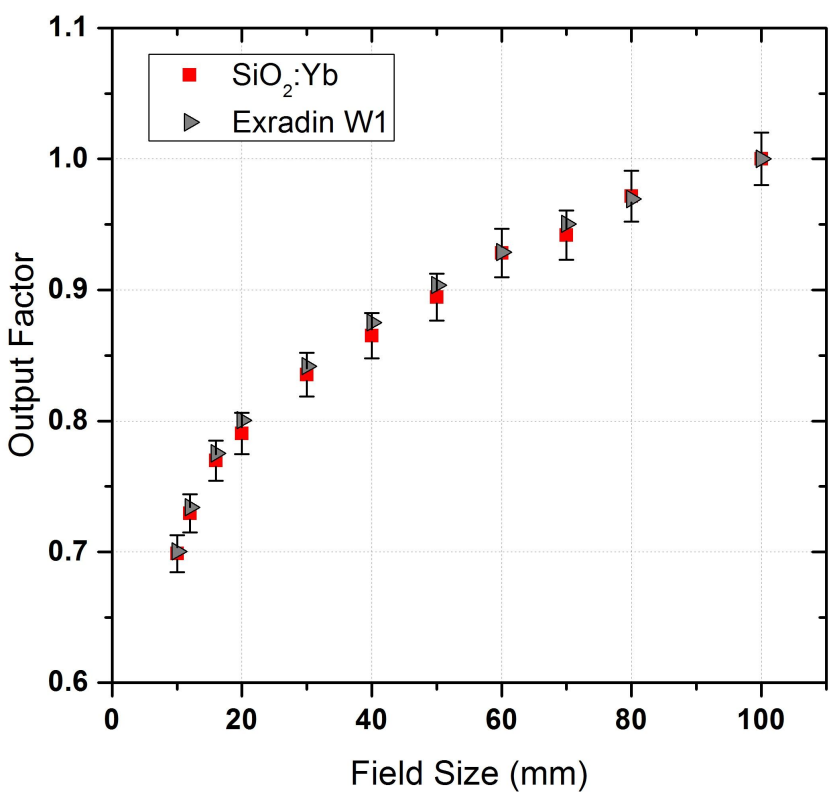

Figure 5. Output Factors (OF) of a $6 \mathrm{MV}$ X-ray FFF beam measured with the Yb-doped silica fiber and compared with the results of the Exradin W1 plastic scintillator. 
It is worth noting that the Exradin W1 proved to be a reliable tool for small field dosimetry applications such as RT beams commissioning and quality checks ${ }^{1,29,30)}$ thanks to its water-equivalence. However, the approach adopted for stem effect correction is very sensitive to the irradiation set $u^{31-34)}$.

By contrast, Yb-doped silica optical fibers are not tissue equivalent, but they enable the complete suppression of the stem effect simply by optical filtering, without the need of any specific calibration procedure.

\section{CONCLUSIONS}

The results of this study demonstrated that the drawback due to the stem effect in Yb-doped silica optical fibers can be managed in a simple but effective way by optical filtering. The various tests carried out using small radiation field sizes and the most challenging irradiation geometries stated the robustness of the system in complex dosimetric scenarios. In fact, the response of the $\mathrm{Yb}$-doped fibers was unaffected by the irradiation set up resulting independent of its orientation inside the radiation field, the angular beam incidence, the length of optical fiber directly irradiated, and of previously absorbed doses.

These features, together with the accuracy and precision achieved by Yb-doped fibers in relative dose assessments make the device promising for in-vivo dosimetry studies of radiotherapy treatments characterized by intensity modulation and steep dose gradients.

\section{REFERENCES}

[1] Das, I. J., Morales, J. and Francescon, P., "Small field dosimetry: What have we learn?," AIP Conf. Proc. 1747, 060001 (2016).

[2] Khan, F.M., [The physics of radiation therapy], Lippincott Williams \&Wilkins, Philadelphia (2003).

[3] Veronese, I., De Martin, E., Martinotti, A.S., et al., "Multi-institutional application of Failure Mode and Effects Analysis (FMEA) to CyberKnife Stereotactic Body Radiation Therapy (SBRT)," Radiation Oncology 10:132 (2015).

[4] Begnozzi, L., Cantone, M.C., Longobardi, B.,et al., "Prospective approaches for risk analysis in modern radiotherapy: the Italian experience and the contribution of the medical physicists," Radioprotection 49, 43-47 (2014).

[5] Giardina, M., Cantone, M.C., Tomarchio, E., et al., "A review of healthcare failure mode and effects analysis (HFMEA) in radiotherapy," Health Physics 111, 317-326 (2016).

[6] Gallo, S., Iacovello, G., Bartolotta, A., et al., "ESR dosimeter material properties of phenols compound exposed to radiotherapeutic electron beams," Nucl. Instr. Meth. B, 407, 110-117 (2017).

[7] Marrale, M., Collura, G., Gallo, S., et al., "Analysis of spatial diffusion of ferric ions in PVA-GTA gel dosimeters through magnetic resonance imaging," Nucl. Instr. Meth. B, 396, 50-55 (2017).

[8] Gambarini, G., Veronese, I., Bettinelli, L., et al., "Study of optical absorbance and MR relaxation of Fricke xylenol orange gel dosimeters," Radiat. Meas. , doi: 10.1016/j.radmeas.2017.03.024 (2017).

[9] Kron, T., Lehmann, J. and Greer, P. B., "Dosimetry of ionising radiation in modern radiation oncology", Phys Med. Biol. 61 R167-205 (2016).

[10]Mones, E., Veronese, I., Moretti, F., et al., "Feasibility study for the use of Ce3+-doped optical fibres in radiotherapy," Nucl. Instrum. Methods. Phys. Res. A 562, 449-455 (2006).

[11] Veronese, I., Fasoli, M., Martini, M., et al., "Phosphorescence of $\mathrm{SiO} 2$ optical fibers doped with Ce3+ ions," Phys. Status Solidi c 4, 1024-1027 (2007).

[12] Mones, E., Veronese, I., Vedda, A., et al., "Ce-doped optical fiber as radioluminescent detector in radiotherapy," Radiat Meas. 43, 888-892 (2008).

[13] Veronese, I., Cantone, M.C., Chiodini, N., et al. "Feasibility study for the use of cerium-doped silica fibers in proton therapy," Radiat. Meas. 45, 635-639 (2010). 
[14]O'Keeffe, S., McCarthy, D., Woulfe, P., et al., "A review of recent advances in optical fibre sensors for in vivo dosimetry dosimetry during radiotherapy,” Br. J. Radiol. 88:20140702 (2015).

[15] Nascimento, L.F., Vanhavere, F., Boogers, E., et al., "Medical dosimetry using RL/OSL prototype," Radiat. Meas. 71, 359-363 (2014).

[16]Beddar, A.S., "Plastic scintillation dosimetry and its application to radiotherapy," Radiat. Meas. 41 S124-S133 (2007).

[17] Beddar, S. and Beaulieu, L., [Scintillation Dosimetry], CRC Press Taylor \&Francis Group, Boca Raton (2016).

[18]Beddar, A. S., Mackie, T. R. and Attix, F. H., "Cerenkov light generated in optical fibres and other light pipes irradiated by electron beams," Phys. Med. Biol. 37, 925-35 (1992).

[19] Carrasco, P., Jomet, N., Jordi, O., et al., "Characterization of the Exradin W1 scintillator for use in radiotherapy," Med.Phys. 42, 297-304 (2015).

[20] Veronese, I., Cantone, M.C., Catalano, M., et al., "Study of the radioluminesence spectra of doped silica optical fiber dosimeters for stem effect removal," J. Phys. D Appl. Phys. 46: 015101 (2013).

[21] Veronese, I., Cantone, M.C., Chiodini, N., et al., "The influence of the stem effect in Eu-doped silica optical fibers," Radiat Meas. 56, 316-319 (2013).

[22] Liu, P.Z.Y., Suchowerska, N., Lambert, J., et al., "Plastic scintillation dosimetry: comparison of three solutions for the Cerenkov challenge," Phys. Med. Biol. 56, 5805-5821 (2011).

[23] Clift, M.A., Johnston, P.N. and Webb, D.V., "A temporal method of avoiding the Cerenkov radiation generated in organic scintillator dosimeters by pulsed mega-voltage electron and photon beams" Phys. Med. Biol. 47, 1421-1433 (2002).

[24] Frelin, A.M., Fontbonne, J.M., Ban, G., et al., "Spectral discrimination of Cerenkov radiation in scintillating dosimeters," Med. Phys. 32 3000-3006 (2005).

[25] Veronese, I., De Mattina, C., Fasoli, M., et al., "Infrared Luminescence for Real Time Ionizing Radiation Detection," Appl. Phys. Lett. 105:061103 (2014).

[26] Veronese, I., De Mattia, C., Fasoli, M., et al., "Role of Optical Fiber Drawing in Radioluminescence Hysteresis of Yb-Doped Silica," J. Phys. Chem. C 119, 15572-15578 (2015).

[27] Veronese, I., Chiodini, N., Cialdi, S., et al., "Realt-time dosimetry with Yb-doped silica optical fibres,".Phys. Med. Biol. 62, 4218-4236 (2017).

[28] Vedda, A. Chiodini, N., Di Martino, D., et al., "Ce3+-doped optical fibers for remote radiation dosimetry," Appl. Phys. Lett. 85, 6536 (2004).

[29] Francescon, P., Beddar, S., Satariano, N., et al., "Variation of k Q clin , Q msr f clin , f msr for the small-field dosimetric parameters percentage depth dose, tissue-maximum ratio, and off-axis ratio," Med. Phys. 41, 101708 (2014).

[30] Francescon, P., Kilby, W., Noll, J. M., et al., "Monte Carlo simulated corrections for beam commissioning measurements with circular and MLP shaped fields on the CyberKnife M6 system: a study including diode, microchamber, point scintillator, and syntetic microdiamond detectors, "Phys. Med. Biol. 621076 (2017).

[31] Morin, J., Béliveau-Nadeau, D., Chung, E., et al., "A comparative study of small field total scatter factors and dose profiles using plastic scintillation detectors and other stereotactic dosimeters: The case of the CyberKnife," Med. Phys. 40, 011719 (2013).

[32] Masi, L., et al, "CyberKnife beam output factor measurements: A multi-site and milti-detector study," Phys. Med. 32, 1637-43 (2016).

[33] Beierholm, A. R., Behrens, C. F. and Andersen, C. E., "Dosimetric characterization of the Exradin W1 plastic scintillator detector through comparison with an in-house developed scintillator system, " Radiat. Meas. 69 5056 (2014)

[34] Papaconstadopoulos, P., Archambault, L. and Seuntjens, J., "Experimental investigation on the accuracy of plastic scintillators and of the spectrum discrimination method in small photon fields," Med. Phys. 44, 654-664 (2017). 